\title{
PROCESOS ELECTORALES RECIENTES Y REPRESENTATIVIDAD DE LOS PARTIDOS: EL CASO CENTROAMERICANO
}

\author{
Manuel Rojas Bolaños*
}

Después de una etapa marcada por profundos conflictos políticos, que en algunos casos llevó a confrontaciones armadas de gran envergadura, las sociedades centroamericanas parecen encaminarse por el sendero de la reconciliación nacional y la democracia política. Aunque la mayoría de los problemas que provocaron los conflictos no han sido resueltos, existe la esperanza de que puedan ser enfrentados exitosamente en un clima de paz y democracia.

En el plano institucional las jóvenes democracias centroamericanas muestran avances y retrocesos. Desde mediados de la década pasada se han venido celebrando regularmente elecciones en todos los países; sin embargo, los partidos políticos tienen dificultades para alcanzar grados aceptables de legitimidad, que les permita desarrollarse como adecuados instrumentos para la agregación y representación de intereses, en un contexto marcado por la reestructuración del Estado y la sociedad.

En este trabajo se examinan los resultados de las elecciones realizadas entre 1993 y 1996 en cuatro de los cinco países de la región, en los aspectos relativos a la situación de los partidos y de los incipientes sistemas de partidos. Los países analizados son Guatemala, El Salvador, Honduras y Costa Rica. Se busca identificar algunas de las similitudes y diferencias entre la situación de los partidos en países donde el conflicto llevó la confrontación política a extremos, y en países en donde la estabilidad, cuando menos relativa, ha sido la tónica. Asimismo se examinan las tendencias y los posibles escenarios.

\section{ELECCIONES}

Como puede observarse en el cuadro $\mathrm{N}^{\circ} 1$, en todos los países centroamericanos se han venido realizando regularmente elecciones desde principios de los años ochenta. Sin embargo, es posible establecer una división entre aquellos

(*) Sociólogo político costarricense, investigador del Programa FLACSO-Costa Rica y profesor de la Maestría en Ciencias Políticas de la Universidad de Costa Rica. En esta ponencia se revisan algunas de las ideas expresadas en el trabajo "Consolidar la democracia en Centroamérica: una ardua tarea", en Tangermann, Klaus D., compilador, Ilusiones y dilemas: la democracia en Centroamérica. San José: Programa FLACSO-Costa Rica/BUNSTIFT e. V., 1995. E-mail: rbolanos@cariari.ucr.ac.cr procesos realizados antes de los acuerdos de Esquipulas II y los de los años posteriores. En efecto, la mayoría de los procesos efectuados antes de dichos acuerdos, debido al pasado de exclusión política extendida y a los conflictos internos, solamente fueron democráticos en la fachada, o si se quiere, siguiendo la formulación de Guy Hermet (1), fueron elecciones de "pluripartidismo excluyentista". Sólo en Costa Rica, realmente, no hubo exclusión, y en menor medida, en Honduras, que había iniciado a principios de la década un proceso de apertura democrática, después de un período de autoritarismo militar; pero cuya situación política estaba muy marcada por los acontecimientos del período anterior y por la polarización ocurrida en la región. En los demás países estuvieron fuera del juego electoral importantes fuerzas políticas: en sociedades como la salvadoreña y la guatemalteca, las fuerzas que cuestionaban la hegemonía de los grupos dominantes tradicionales; en Nicaragua, buena parte de los grupos que se oponían al proceso revolucionario comandado por los sandinistas.

Los procesos electorales realizados antes de los acuerdos de Esquipulas II, ofrecían entonces limitadas posibilidades de participación, por lo menos en dos de los países analizados. Ciertamente, como lo indica Torres Rivas (2), en dichos procesos participó un número relativamente elevado de partidos políticos y no fueron en esencia procesos fraudulentos, además de que buena parte de los candidatos eran políticos civiles; pero también es cierto que no existían suficientes garantías para una participación ciudadana extendida y segura. Aunque no existe una relación causa efecto, la ausencia en el escenario electoral de importantes fuerzas políticas posiblemente contribuyó a los altos índices de abstencionismo observados en algunos de esos procesos electorales. Por ejemplo, en Guatemala, en las elecciones del 3 noviembre de 1985 el abstencionismo fue del 31,0 por ciento; pero aumentó al 35 por ciento en la segunda vuelta (3). No hay datos confiables para las elecciones presidencia-

(1) Hermet, Guy, "Las elecciones en los regímenes autoritarios: bosquejo de un marco de análisis", en Hermet, Guy et. al., ¿Para qué sirven las elecciones?. México: Fondo de Cultura Económica, 1986, 13.

(2) "Imágenes, siluetas, formas en las elecciones centroamericanas: las lecciones de la década", Polémica, Nos. 14-15, Segunda Epoca (mayo-diciembre 1991), 4.

(3) Rosada, Héctor, "Elecciones y democracia en América Latina. Guatemala 1990-1991", en Cerdas, Rodolfo; Rial, Juan y Zovatto, Daniel, editores, Elecciones y democracia en América Latina, 1988-1991: una tarea inconclusa. San José: Instituto 
les de 1984 en El Salvador; sin embargo, en las elecciones legislativas de 1985, el abstencionismo fue aproximadamente el 41 por ciento y en las elecciones presidenciales de 1989 , anteriores a la firma de los acuerdos de paz, el abstencionismo alcanzó el 54,6 por ciento. de los votantes con carnet (4).

\section{Cuadro 1 \\ ELECCIONES REALIZADAS EN CENTROAMÉRICA ENTRE 1980 Y 1994}

\begin{tabular}{|c|c|}
\hline País & Tipo de elección y fecha \\
\hline Guatemala & $\begin{array}{l}\text { Generales } 07 / 03 / 1982 \\
\text { Asamblea Constituyente } 10 / 06 / 1984 \\
\text { Generales }\left(1^{\mathrm{a}} \text { vuelta) } 03 / 11 / 1985\right. \\
\text { Presidenciales }\left(2^{\mathrm{a}} \text { vuelta) } 08 / 12 / 1985\right. \\
\text { Corporaciones municipales } 24 / 04 / 1988 \\
\text { Generales ( } 1^{\mathrm{a}} \text { vuelta) } 11 / 11 / 1990 \\
\text { Presidenciales ( } 2^{\mathrm{a}} \text { vuelta) 06/01/1991 } \\
\text { Parlamentarias extraordinarias } 14 / 08 / 1994 \\
\text { Generales } 12 / 11 / 95\left(1^{\mathrm{a}} \text { vuelta) }\right. \\
\text { Presidenciales } 07 / 01 / 96\left(2^{\mathrm{a}} \text { vuelta) }\right. \\
\end{array}$ \\
\hline El Salvador & $\begin{array}{l}\text { Asamblea Constituyente } 28 / 03 / 1982 \\
\text { Presidenciales ( } 1^{\text {a }} \text { vuelta) } 25 / 03 / 1984 \\
\text { Presidenciales ( } 2^{\mathrm{a}} \text { vuelta) } 06 / 05 / 1984 \\
\text { Parlamentarias y municipales } 31 / 03 / 1985 \\
\text { Parlamentarias y municipales } 20 / 03 / 1988 \\
\text { Presidenciales } 19 / 03 / 1989(1) \\
\text { Parlamentarias y municipales } 10 / 03 / 1991 \\
\text { Presidenciales 20/03/1994 ( } 1^{\mathrm{a}} \text { vuelta) } \\
\text { Presidenciales 24/04/1994 (2 } 2^{\mathrm{a}} \text { vuelta) }\end{array}$ \\
\hline Honduras & $\begin{array}{l}\text { Asamblea Constituyente } 24 / 04 / 1980 \\
\text { Generales } 29 / 11 / 1981 \\
\text { Generales } 24 / 11 / 1985 \\
\text { Generales } 26 / 11 / 1989 \\
\text { Generales } 28 / 11 / 1993 \\
\end{array}$ \\
\hline Nicaragua & $\begin{array}{l}\text { Generales y Asamblea Constituyente 04/11/1984 } \\
\text { Generales 25/02/1990 } \\
\text { Regionales Costa Atlántica 27/02/1994 }\end{array}$ \\
\hline Costa Rica & $\begin{array}{l}\text { Generales } 07 / 02 / 1982 \\
\text { Generales } 02 / 02 / 1986 \\
\text { Generales } 04 / 02 / 1990 \\
\text { Generales } 06 / 02 / 1994\end{array}$ \\
\hline
\end{tabular}

(1) Puesto que el candidato de ARENA, Alfredo Cristiani, obtuvo el 50 por ciento de la votación, la segunda vuelta no se realizó.

Fuente: FLACSO, Perfil Estadístico Centroamericano. San José: FLACSO, 1992 y ss.; Cerdas, $R$. et al., editores, Una tarea inconclusa. Elecciones y democracia en América Latina, 1988-1991. San José: IIDH/CAPELFriedrich Naumann Stiftung, 1992, 708 y ss.

No obstante, dichos procesos tenían un carácter dual, porque si bien es cierto que existía un afán de "...reconstituir, legitimar, normalizar el poder político", como lo señalaba Torres Rivas (5), sin desmontar la estructura autoritaria, dada la presión internacional, la situación interna y las pugnas dentro del mismo grupo gobernante, no pocos efectos "democratizantes" tuvieron sobre la sociedad. Se crearon instituciones encargadas de dirigir y verificar los procesos, se dictaron códigos y otras regulaciones, y se permitió la presencia de observadores extranjeros calificados. Como lo ha indicado Linz (6), las consecuencias de las

Interamericano de Derechos Humanos/CAPEL/Friedrich Naumann Stiftung, 1992, 88. El abstencionismo se refiere e al porcentaje de los electores inscritos.

(4) Eguizábal, Cristina, "El Salvador: procesos electorales y democratización", en Cerdas, Rodolfo; Rial, Juan y Zovatto, Daniel, editores, op. cit., 58; FLACSOPrograma El Salvador, El proceso electoral 1994. San Salvador: FLACSO-El Salvador, 1995,37 y 174.

(5) Torres Rivas, 2

(6) Linz, Juan J., "Funciones y disfunciones de las elecciones no competitivas: los sistemas autoritarios y totalitarios", en Hermet, Guy et. ali., ¿Para qué sirven las elecciones? México: Fondo de Cultura Económica, 1986, 92. elecciones sobre el sistema político pueden ser diferentes a las intenciones de los dirigentes o a las motivaciones de los electores. En ese sentido, los procesos electorales de los años ochenta en Centroamérica deben verse, como lo reconoce el mismo Torres Rivas (7), como

“...un primer paso, en sociedades donde la violencia impone su dinámica, para acortar aquella distancia e inaugurar con todas sus deficiencias una de las tantas formas de participación política, para empezar a restituir a la conciencia ciudadana la sensación de construir la legitimidad del poder."

Los procesos electorales de los años noventa indican, sin embargo, que pese a los esfuerzos realizados para depurar y modernizar los mecanismos de la democracia política, entre ellos los sistemas electorales (8), el poder político no termina de legitimarse. En Guatemala y El Salvador sigue siendo elevado el porcentaje de abstencionismo, como índice de participación en la contienda electoral; en Honduras creció el abstencionismo en las elecciones presidenciales realizadas en noviembre de 1993 con respecto a las de cinco años atrás, y solamente en Costa Rica, cuyas instituciones políticas han conservado la estabilidad por más de cuarenta años, el abstencionismo se mantuvo dentro de sus límites históricos.

\section{Cuadro 2 \\ PORCENTAJE DE ABSTENCIONISMO \\ EN LOS ÚLTIMOS PROCESOS ELECTORALES (ELECCIONES PRESIDENCIALES)}

\begin{tabular}{lc}
\hline Países & $\begin{array}{c}\text { Porcentaje de } \\
\text { abstencionismo }\end{array}$ \\
\hline Costa Rica, 1994 & 18,8 \\
\hline El Salvador, 1994 & \\
Primera vuelta & 47,3 \\
Segunda vuelta & 54,1 \\
\hline Honduras, 1993 & 41,0 \\
\hline Guatemala, 1995-1996 & \\
Primera vuelta & 56,0 \\
Segunda vuelta & 63,1
\end{tabular}

Fuente: organismos electorales y prensa.

\section{LOS PARTIDOS POLITICOS}

Más allá de los problemas técnicos, los resultados de las elecciones y, en general, el futuro de la democratización política, tiene que ver en buena medida con la situación de los instrumentos esenciales de la participación política, es decir, los partidos, pues teóricamente corresponde a ellos el papel de intermediación entre el Estado y la sociedad civil. Como lo señaló hace mucho tiempo Duverger (9), hay una relación intrínseca entre la aparición de los partidos políticos, el parlamentarismo y el desarrollo de los procedimientos electorales.

En los regímenes democráticos liberales, entonces, los mecanismos idóneos para la expresión y la representación de intereses dentro de la institucionalidad del Estado, son los partidos políticos; es a través de ellos que se logra la re-

(7) Torres Rivas, 3

(8) En Guatemala, El Salvador, Honduras y Nicaragua se aprobaron leyes electorales que buscaban establecer condiciones mínimas para una participación electoral de una mayor amplitud.

(9) Duverger, Maurice, Sociología política. Barcelona: Ediciones Ariel, 1972, 307. 
lación entre electores y representantes (10); al menos esa ha sido la experiencia de las democracias del norte. De acuerdo con el modelo de democracia liberal, la existencia de elecciones libres y de un respeto extendido para las libertades individuales es fundamental para el asentamiento de un régimen democrático, pero también lo es la existencia de un sistema de partidos políticos funcionando normalmente.

En este aspecto la situación de Centroamérica ofrece algunos contrastes. En tres de los países analizados el número de partidos activos es relativamente elevado. Algunos son partidos con varias décadas de existencia, que lograron sobrevivir en las difíciles condiciones imperantes, muchas veces en asocio con los regímenes. Por ejemplo, en Guatemala y El Salvador siguió funcionando un buen número de partidos políticos durante el período autoritario, varios de ellos integrados o apoyados por los militares. Por supuesto, en la mayoría de los casos se trataba de partidos que contribuían a mantener una imagen de "legitimidad" del régimen, a cambio de unos cuantos asientos en los parlamentos o de algunos cargos secundarios en el gobierno; la actividad partidaria, por tanto, estaba generalmente reducida a una limitada participación en los procesos electorales. Salvo contadas excepciones, la mayoría de estos partidos ha continuado funcionando en la nueva etapa de apertura democrática, a la par de otros de reciente creación, que surgieron, como en El Salvador, después de los acuerdos de paz.

Sin embargo, quizá con las excepciones de Costa Rica y Honduras, no es posible encontrar partidos, y mucho menos sistemas de partidos, que cumplan con buena parte de los requisitos del modelo occidental. La mayor parte de ellos están más cercanos a la definición mínima de un partido ofrecida por Sartori: "Un partido es cualquier grupo político que se presenta a elecciones y que puede colocar mediante elecciones a sus candidatos en cargos públicos" (11). En efecto, la mayoría de los viejos partidos, así como muchos de los de reciente aparición, son organizaciones débiles, que carecen de una estructura extendida a nivel nacional, que movilizan con dificultades a una masa importante de electores y cuya actividad está casi totalmente restringida al plano electoral. Partidos muy ligados a grupos o sectores sociales específicos, carentes de la autonomía que les permitiría la agregación y representación de intereses diversos.

Como puede observarse en el Cuadro $\mathrm{N}^{\circ} 3$, el número de partidos participantes en las últimas elecciones en cada país es elevado; sin embargo, muchos son partidos pequeños, poco importantes desde el punto de vista "sistémico". Por ejemplo, en la primera vuelta de las elecciones presidenciales de Guatemala, celebradas en noviembre de 1995 , participaron 28 partidos, varios en coalición, y se presentaron 19 candidatos a la presidencia de la República; además de un número elevado de candidatos a los 80 escaños del Congreso, a los 300 consejos municipales y a los 20 asientos en el Parlamento Centroamericano.

(10) Cuando menos esta ha sido la regla; sin embargo, no puede dejar de mencionarse la existencia de una crisis de la representación política, que pone en entredicho la efectividad de los mecanismos que han sido preponderantes en el juego político dentro de las democracias liberales, entre ellos el partido político.

(11) Sartori, Giovanni, Partidos y sistemas de partidos, 1. Madrid: Alianza Universidad, 1987, 92.

\section{Cuadro 3 \\ CENTROAMÉRICA, PARTIDOS PARTICIPANTES EN LA ÚLTIMA ELECCIÓN PRESIDENCIAL, POR PAÍS}

\begin{tabular}{|c|c|c|}
\hline País & Ã̃o & Partidos participantes \\
\hline $\begin{array}{l}\text { Guatemala(1) } \\
\text { (primera vuelta) }\end{array}$ & 1995 & $\begin{array}{l}\text { Partido de Avanzada Nacional } \\
\text { Alianza Nacional(2) } \\
\text { Frente Democrático Nueva Guatemala } \\
\text { Frente Republicano Guatemalteco } \\
\text { Partido Libertador Progresista } \\
\text { Unión Democrática }\end{array}$ \\
\hline $\begin{array}{l}\text { El Salvador } \\
\text { (primera vuelta) }\end{array}$ & 1994 & $\begin{array}{l}\text { Alianza Republicana Nacionalista } \\
\text { Coalición integrada por el FMLN, la } \\
\text { Convergencia Democrática y el } \\
\text { Movimiento Nacionalista Revolucionario(3) } \\
\text { Demócrata Cristiano } \\
\text { Conciliación Nacional } \\
\text { Movimiento Auténtico Cristiano } \\
\text { Movimiento de Solidaridad Nacional } \\
\text { Movimiento de Unidad }\end{array}$ \\
\hline Honduras & 1993 & $\begin{array}{l}\text { Partido Nacional } \\
\text { Partido Liberal } \\
\text { Partido de Innovación y Unidad } \\
\text { Partido Demócrata Cristiano de Honduras }\end{array}$ \\
\hline Costa Rica & 1994 & $\begin{array}{l}\text { Partido Liberación Nacional } \\
\text { Partido Unidad Social Cristiana } \\
\text { Partido Fuerza Democrática } \\
\text { Partido Alianza Nacional Cristiana } \\
\text { Partido Unión Generaleña } \\
\text { Partido Nacional Independiente } \\
\text { Partido Independiente }\end{array}$ \\
\hline
\end{tabular}

(1) Solamente se indican los nombres de los partidos que obtuvieron más de 50.000 votos en dichas elecciones. Una segunda vuelta se realizó el 7 de enero de 1996, con la participación de los candidatos del PAN y del FRG.

(2) Coalición integrada por la Democracia Cristiana Guatemalteca, la Unión del Centro Nacional y el Partido Socialista Democrático.

(3) Posteriormente se separaron del FMLN el Movimiento Nacional Revolucionario (MNR), la Expresión Renovadora del Pueblo (ERP) y la Resistencia Nacional (RN), para dar paso a la constitución del Partido Demócrata.

Fuente: organismos electorales y prensa.

Los resultados de las elecciones, como se observa en el Gráfico $\mathrm{N}^{\circ} 1$, muestran un panorama en donde unos pocos partidos controlan el flujo electoral; no obstante, la situación es diferente en los dos países donde la democratización es más reciente que en Honduras y Costa Rica. En efecto, en Guatemala y El Salvador resalta la presencia de un partido fuerte frente a una cierta dispersión de fuerzas; mientras que en Honduras y Costa Rica el electorado se reparte fundamentalmente en dos partidos.

En Guatemala y El Salvador las debilidades y la inestabilidad actual de los partidos son en buena parte el resultado de décadas de represión; sin embargo, no todo es achacable a las circunstancias políticas de esos países en la segunda mitad de este siglo. Hay otros factores en juego que es necesario mencionar, y que también afectan a los partidos de Honduras y Costa Rica. En primer lugar, las difíciles condiciones socioeconómicas así como las necesidades de adaptación de las economías a las nuevas condiciones del mercado mundial, han terminado por conformar una especie de escenario monocromático, que impide a los partidos la elaboración de propuestas programáticas que difieran substancialmente de lo que señalan los organismos multilaterales. Aunque algunos partidos, como el Liberal de Honduras o Liberación Nacional de Costa Rica, han intentado presentar a consideración de los electores programas supuestamente alternativos a la propuesta neoliberal, una vez en el gobierno se han visto obligados a continuar con el ajuste macroeconómico, la apertura comercial y la reducción del aparato estatal, con el consiguiente desencanto de los electores. 


\section{Gráfico 1 \\ RESULTADOS DE LAS ELECCIONES PRESIDENCIALES (PORCENTAJES)}
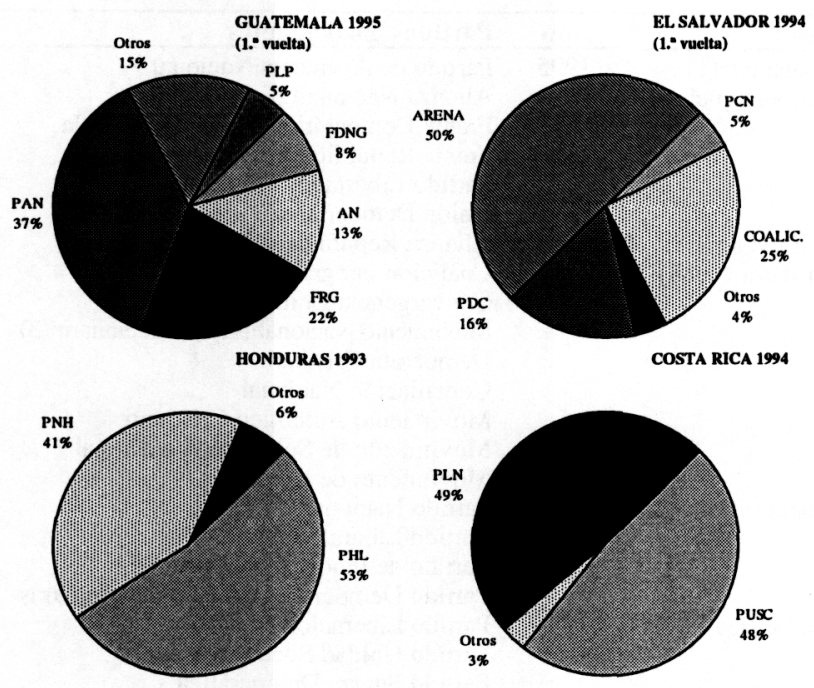

En la práctica, entonces, se han ido borrando las divisiones ideológicas y las viejas identidades partidarias, basadas en planteamientos particulares sobre la economía y el papel del Estado. Aunque todavía subsisten posiciones extremas, las agrupaciones políticas más importantes se han ido moviendo hacia una posición que podría ser calificada como de centro derecha (12). Incluso algunos de los sectores conocidos hasta hace poco por sus planteamientos de izquierda marxista, han empezado a moverse hacia ese centro, asumiendo posiciones a veces calificadas como de "liberalismo social".

El incumplimiento de las promesas de campaña por parte de los gobernantes electos, en los casos de Guatemala y El Salvador, no sólo se refiere al manejo de la economía y al mejoramiento de la situación social de la mayor parte de la población, sino también al mantenimiento de un Estado de derecho y al respeto a los derechos humanos. En la mayoría de los países de Centroamérica esta es todavía una tarea inconclusa.

En segundo lugar, a los viejos problemas sociales no solucionados se han venido a sumar los provocados por el ajuste macroeconómico, lo que complica el panorama. En tres de los cuatro países analizados la pobreza sigue siendo muy elevada; pero la estructura social centroamericana se ha tornado, en general, más compleja, con la aparición o el incremento de nuevos sectores sociales sobre todo en el ámbito urbano, muy desorganizados y marginalmente interesados en la política. En el caso guatemalteco la situación es todavía más difícil, porque que se trata de una sociedad partida en dos, étnicamente hablando. En efecto, de acuerdo con los datos del Censo de 1984, un 44 por ciento de la población es maya; sin embargo, hay quienes afirman que este porcentaje es todavía más elevado. En todo caso, no sólo se trata de una población con lenguas y culturas propias, sino también de una población que ha sufrido segregación social y política. La Revolución de octubre de 1944 intentó integrarla al sistema político en igualdad de condiciones a la población ladina o blanca; pero a partir del golpe de 1954 el terror invadió el

(12) Este movimiento podría ser positivamente evaluado en una situación en donde la polarización social no fuera tan radical. campo guatemalteco y los indígenas han sido uno de los sectores más golpeados por la represión que desde entonces se desató. Para la mayor parte de esta población la participación política ha significado violencia y sufrimiento, lo que ha provocado su marginación. La política es para los ladinos, incluso para un sector de ellos; mientras que los indígenas permanecen en su mayor parte al margen de ese mundo, como lo reflejan los resultados de las elecciones.

En tercer lugar, a la debilidad organizativa y programática que dificulta la construcción de sistemas de partidos relativamente estables, se vienen a agregar factores derivados de la cultura política. En efecto, en la mayoría de los países de Centroamérica se ha desarrollado una cultura de la intolerancia que limita demasiado el juego político, y que termina por convertir las discrepancias en enfrentamientos tipo "juegos suma cero", que no pocas veces finalizan con la eliminación física del adversario. En cuatro de los cinco países de la región el asesinato político ha sido un procedimiento usado con alguna frecuencia, destacándose el caso de Guatemala, donde la "cosecha de violencias" ha sido extremadamente grande (13). La cultura política dominante solamente incluye las prácticas democráticas como excepción, y no como denominador común para la resolución de conflictos; tampoco el respeto extendido a la libertad de pensamiento y organización política. Estos factores se reflejan en la ambivalencia que muchos partidos mantienen en sus relaciones con los otros; tienen dificultades para aceptar a los otros como adversarios en una competencia democrática. La oposición se siente sin garantías y la alternancia es vista por unos y otros, aunque por diferentes razones, como una seria amenaza para vidas y haciendas.

El personalismo es otro factor que conspira contra el fortalecimiento de los partidos. En una cultura política donde el caudillismo ha sido un elemento central, difícilmente se pueden encontrar partidos fuertes que no sean personalistas, es decir, cuya actividad no gire alrededor de una o de unas pocas figuras que encarnan al partido y a su supuesta "ideología". Las plataformas programáticas o los planteamientos ideológicos carecen realmente de importancia. Si bien es cierto que los partidos necesitan liderazgos fuertes, y que en la definición de las preferencias partidarias ese es a veces un factor más importante que las coincidencias con plataformas programáticas o planteamientos ideológicos, en Centroamérica el caudillismo ancestral parece agravar la tendencia, sobre todo en esta era del ajuste, donde las presiones del entorno impiden a los partidos presentar programas que se aparten del llamado consenso de Washington. La imposibilidad de diferenciarse programáticamente, entonces, refuerza el personalismo, inclusive en un país como Costa Rica.

La tradición caudillista ha encontrado su continuidad, en la actual etapa de apertura democrática, en el carácter presidencialista del régimen vigente en todos los países de la región. Por supuesto que es diferente el peso del presidencialismo en un país como Costa Rica, que en contextos de tradición autoritaria. Como lo señala Mainwaring (14), bajo condiciones de-

(13) Al respecto ver, entre otros, Carmarck, Robert M., compilador, Guatemala: cosecha de violencias. San José: FLACSO, 1991.

(14) Mainwaring, Scott, "Presidentialism in Latin America", Latin American Research Review, Vol. XXV, Number I, 1990, 157-179. 
mocráticas, los presidentes tienen mucho más problemas en lograr que sus proyectos se concreten, que en contextos autoritarios. Este es un elemento que hay que tomar en cuenta dentro del análisis; sin embargo, en mayor o menor medida el presidencialismo es otro de los factores que está impidiendo la construcción de estructuras partidarias que sean algo más que meras plataformas electorales. La experiencia reciente muestra como una vez pasadas las elecciones los partidos se diluyen, dejando a los candidatos ganadores libres de cualquier control partidario que les obligue a cumplir programas o compromisos adquiridos durante las campañas electorales. En los períodos entre una y otra elección, generalmente sólo las fracciones parlamentarias se mantienen en actividad; los otros órganos del partido prácticamente desaparecen. Pero como el peso del candidato de turno en la selección de los diputados es muy elevado, las fracciones parlamentarias del partido triunfante en las elecciones son muy sumisas al presidente y casi no ejercen control sobre el ejecutivo. En esas condiciones difícilmente los parlamentos pueden convertirse en los grandes foros de debate de los problemas nacionales. Han perdido la iniciativa, se encuentran semiparalizados y su descrédito es creciente, como lo muestran los sondeos de opinión. El caso extremo es el de Guatemala, donde el abstencionismo alcanzó casi el 80 por ciento en las elecciones parlamentarias de agosto de 1994; pero en mayor o menor grado la situación es similar en todos los países de la región, incluyendo Costa Rica, donde la Asamblea Legislativa ha ido cediendo paulatinamente terreno al ejecutivo. En este aspecto el panorama regional es de deterioro del poder legislativo y de fortalecimiento del ejecutivo.

\section{LAS TENDENCIAS}

Desde el punto de vista de los ciudadanos el escenario no es muy favorable para el desarrollo de los partidos políticos. Los sondeos de opinión indican la existencia de un descontento, cuando menos en tres de los países analizados. Por ejemplo, una encuesta realizada en Guatemala en mayo de 1993 (15), pocos días antes de frustrado autogolpe protagonizado por el entonces Presidente Serrano, muestra un reducido grado de confianza en los partidos políticos: 18 por ciento de los entrevistados. En El Salvador (16), una encuesta realizada por el Instituto Universitario de Opinión Pública (IUDOP), poco antes de las elecciones de marzo de 1994, reveló que más del 50 por ciento de los entrevistados carecía de interés en el proceso político o indicaba un interés marginal. Una encuesta reciente realizada en Costa Rica por la firma UNIMER (17), señaló un importante incremento, en relación a encuestas anteriores, del porcentaje de personas que se declararon "sin partido": 26,6 por ciento. Aproximadamente el 50 por ciento de los que se manifestaron de esa forma indicó una pertenencia anterior a algún partido. Entre las razones de este

(15) Development Associates, Inc., University of Pittsburgh y Asociación de Investigación y Estudios Sociales (ASIES), La cultura democrática de los Guatemaltecos. Guatemala: enero de 1995, 38.

(16) ECA (Estudios Centroamericanos), Año XLIX, No 545-546 (marzo-abril 1994), 276.

(17) UNIMER, Tercera encuesta cuatrimestral de opinión nacional (7-19 de setiembre 1995). San José: UNIMER (mimeógrafo), 48 y ss. "apartidarismo", la más señalada deja entrever frustración con el desempeño de los dirigentes: 49 por ciento (18). Del total de entrevistados, el 43 por ciento manifestó no encontrar diferencias en cuanto a los planteamientos y comportamiento de las dos agrupaciones mayoritarias del país.

\section{Gráfico 2 \\ COMPOSICIÓN ACTUAL DE LOS PARLAMENTOS POR EL NÚMERO DE ESCAÑOS POR PARTIDO}
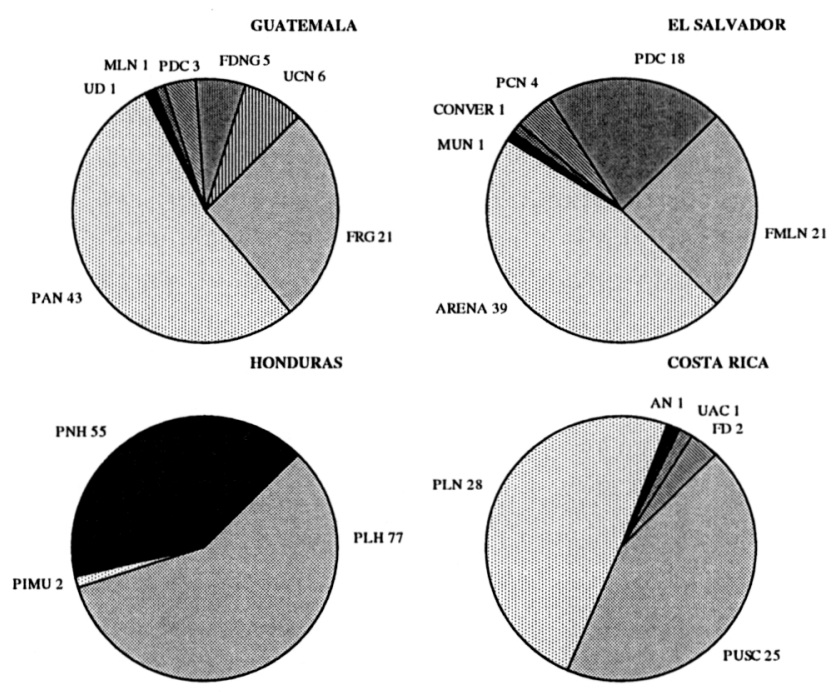

Una encuesta realizada por las unidades de la FLACSO en Guatemala, El Salvador y Costa Rica, a sectores sociales específicos, arrojó resultados similares: 64,4 por ciento de los entrevistados en Guatemala, 59,2 por ciento en El Salvador y 59,3 por ciento en Costa Rica, reaccionaron negativamente a la afirmación "los partidos políticos representan los intereses de los ciudadanos" (19). Pese a las objeciones que se le pueden hacer a las encuestas como instrumentos de acercamiento a la realidad — en este caso a la realidad política-, es indudable que reflejan climas de opinión. En ese sentido, los resultados de este conjunto de encuestas están revelando un sentimiento ciudadano de malestar con los partidos políticos, sobre todo en lo que se refiere a la lejanía entre las preocupaciones cotidianas de los ciudadanos y la acción de los dirigentes.

Pero las críticas habría que tomarlas con un grano de sal. Petra Bendel (20), indica que muchas de las críticas que se hacen a los partidos políticos en Centroamérica, parten de visiones que toman modelos de partido propios de realidades que poco o nada tienen que ver con el contexto regional. Según esta autora (21), una mejor comprensión de lo que pasa en la región en cuanto a partidos políticos y sistemas de partidos se refiere, podría lograrse siguiendo en líneas generales lo que Sartori denomina "comunidades políticas fluidas de par-

(18) "Se siente traicionado por los dirigentes".

(19) Rojas Bolaños, Manuel, Ajuste, sectores populares y gobernabilidad. San José: Programa FLACSO-Costa Rica, 1995, 111 y ss. En cada país se escogió una muestra de 1.250 individuos de sectores campesinos, de trabajadores urbanos por cuenta propia y de empleados públicos de salud y educación.

(20) Bendel, Petra, Democracia y partidos políticos en América Central. Heidelberg: Institut Für Poltische Wissenschaft, Universitat Heidelberg, 1993, 3 y ss.

(21) Bendel, Petra, "Partidos políticos y sistemas de partidos en Centroamérica", en Nohlen, Dieter, editor, Elecciones y sisemas de partidos en América Latina. San José: IIDH-CAPEL, 1993, 326 y ss. 
tidos" (22). De esa manera, la situación centroamericana, con la excepción de Costa Rica y Honduras, podría describirse como en evolución, de una situación poco estructurada, no competitiva, hacia sistemas de partidos estables y competitivos, que podría ser bipartidistas o multipartidistas, dependiendo de las particularidades nacionales y, por supuesto, de la influencia de los sistemas electorales (23).

De acuerdo con este planteamiento, en dos de los países analizados en este trabajo aparentemente se está evolucionando hacia a un agrupamiento de fuerzas, que posiblemente llevará a la conformación de sistemas de dos o tres partidos grandes (24); mientras que en Honduras y Costa Rica se mantiene un esquema bipartidista. Así parece corroborarlo la distribución actual de escaños en los parlamentos, como puede observarse en el Gráfico $\mathrm{N}^{\circ} 2$. Como fue señalado, en Guatemala, pese al gran número de partidos inscritos, el congreso está controlado prácticamente por dos partidos; en El Salvador, el Partido ARENA controla el 46 por ciento de los escaños, mientras que el FMLN y la Democracia Cristiana en conjunto controlan un porcentaje similar (25); en Honduras el Partido Nacional y el Partido Liberal se distribuyen el 98,5 por ciento de los escaños, y en Costa Rica dos grandes agrupaciones se reparten más del 90 por ciento de los diputados (26).
Sin embargo, el destino de esta evolución, en los casos del Guatemala y El Salvador —así como el mantenimiento del bipartidismo existente en Honduras y Costa Rica-, depende de la posibilidad de introducir modificaciones que les permitan captar y procesar adecuadamente las demandas de la sociedad, lo que implica formas de organización mucho más abiertas, que faciliten una interacción constante y fluida con la sociedad y con los movimientos que ocurren dentro de ella, a fin de poder plantear plataformas acordes con los tiempos que vivimos de globalización y de espacio cibernético, que a la vez abran vías para la solución a los problemas de pobreza y exclusión social en que se encuentran las mayorías. Sólo en esa medida podrán aspirar a representar a amplios sectores de la sociedad y cumplir su cometido como instrumentos de democratización.

Sin embargo, las condiciones no son favorables para cambios radicales en estos aspectos en el corto plazo. Como fue mencionado, factores internos y externos limitan considerablemente las posibilidades de maniobra, más allá de las intenciones de los dirigentes o las propuestas de los analistas. Por esa razón, es de esperar la prolongación, por unos años más, del déficit observado de representatividad y, por tanto, de legitimidad de los partidos políticos en Centroamérica.

\section{RESUMEN}

En este trabajo se examinan los resultados de las elecciones realizadas entre 1993 y 1996 en cuatro de los cinco países de la Región, en los aspectos relativos a la situación de los partidos y de los incipientes sistemas de partidos. Se busca identificar algunas de las siomilitudes y diferencias entre la situación de los partidos en países donde el conflicto llevó la confrontración política a extremos, y en países en donde la estabilidad, cuando menos relativa, ha sido la tónica. Asimismo se examinan las tendencias y los posibles escenarios.

\section{ABSTRACT}

In this article, the author deals with elections results in Guatemala, El Salvador, Costa Rica and Honduras between 1993 and 1996. The principal issues are the relationship between election results and party systems in a comparativa perspective in the countries that experience civil conflict and in the countries without civil conflict. Anothe issues dealed are the trends and the future scenes for the items. 